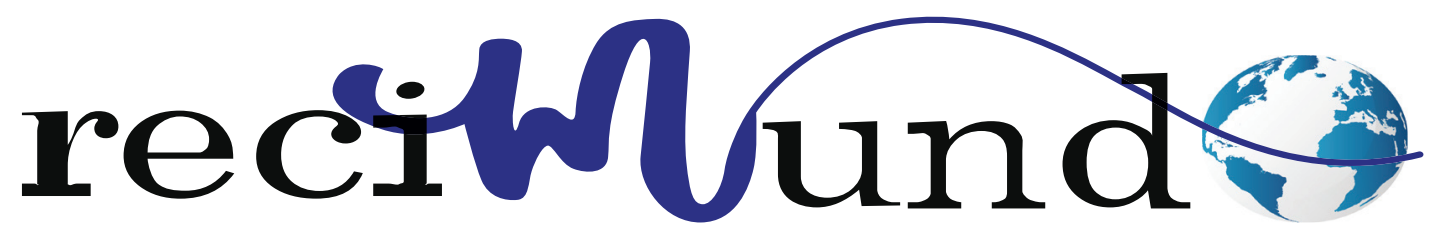

Revista Científica Mundo de la Investigación y el Conocimiento

DOI: 10.26820/recimundo/4.(3).julio.2020.42-52

URL: http://recimundo.com/index.php/es/article/view/847

EDITORIAL: Saberes del Conocimiento

REVISTA: RECIMUNDO

ISSN: 2588-073X

TIPO DE INVESTIGACióN: Artículo de Revisión

CÓDIGO UNESCO: 3314 Tecnología Medica

PAGINAS: 42-52

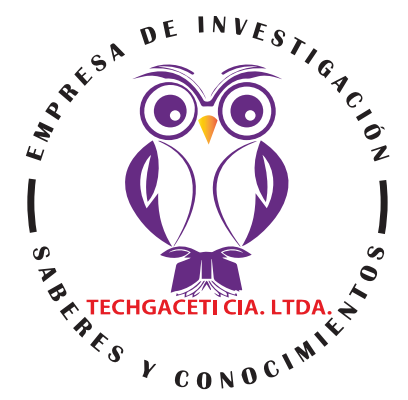

\title{
Destete de la ventilación mecánica, Guayaquil 2019
}

Weaning from mechanical ventilation, Guayaquil 2019

Desmame da ventilação mecânica, Guayaquil 2019

\begin{abstract}
Raúl Germán Castro García; Manuel Jesús Pazmiño Chancay2; María Rosa Zambrano Iza3;
\end{abstract} Erick jhon Sánchez Acuña ${ }^{4}$

\section{RECIBIDO: 10/04/2020 ACEPTADO: 26/05/2020 PUBLICADO: 01/07/2020}

1. Magister en Diseño Curricular; Diploma Superior en Docencia Universitaria; Diploma Superior en Diseño Curricular por Competencias; Licenciado en Terapia Respiratoria; Tecnólogo Medico en Terapia Respiratoria; Docente de las Carrera de Terapia Respiratoria, Facultad de ciencias médicas. Universidad de Guayaquil; Guayaquil, Ecuador; castrogarciaraul50@ gmail.com; (D) https://orcid.org/0000-0003-4315-2031

2. Magister en Emergencias Médicas; Magister en Medicina Forense; Especialista en Atención Primaria de la Salud; Diploma Superior en Salud Ocupacional; Médico; Universidad de Especialidades Espíritu Santo; Samborondón, Ecuador; pazmiño_1982@hotmail.com; (iD https://orcid.org/0000-0001-8712-8442

3. Magister en Diseño Curricular; Diploma Superior en Diseño Curricular por Competencias; Tecnóloga Medica en Terapia Ocupacional; Docente de la Carrera de Terapia Ocupacional, Facultad de ciencias Médicas. Universidad de Guayaquil; Guayaquil, Ecuador; maria.zambranoi@ug.edu.ec; (D) https://orcid.org/0000-0002-7497-7151

4. Médico; Investigador Independiente; Guayaquil, Ecuador; erickj2385@hotmail.com; (D) https://orcid.org/0000-0003-25303879

\section{CORRESPONDENCIA}

Raúl German Castro García

castrogarciaraul50@gmail.com

Guayaquil, Ecuador

() RECIMUNDO; Editorial Saberes del Conocimiento, 2020 


\section{RESUMEN}

El destete de la ventilación mecánica es el proceso de desconexión o retirada del soporte ventilatorio artificial. No existen criterios absolutos para prevenir un destete fallido o exento de complicaciones, pero es aconsejable utilizar protocolos con sus predictores y criterios de destete para minimizar consecuencias indeseadas. Como objetivo se propuso establecer los conocimientos, actitudes y prácticas de los profesionales del cuidado respiratorio en el destete de la ventilación mecánica en centros públicos y privados de la ciudad de Guayaquil. El diseño de la investigación fue cuantitativo, no experimental, transversal, observacional, con enfoque descriptivo. La muestra estuvo constituida por 214 terapistas respiratorios de 6 clínicas privadas y 8 hospitales públicos de la ciudad de Guayaquil a los cuales se les aplico una encuesta de dieciséis preguntas como instrumento de recolección de datos. Como resultado se obtuvo que $58 \%$ de los terapistas usan protocolos de destete, asegurando que el $98 \%$ de los casos es indicado por el médico y solo el $2 \%$ realizan el destete por decisión propia. En cuanto a la evaluación de la hemodinámica del paciente, 88,3\% de los terapistas respiratorios expresaron usar el índice de Tobin, cociente frecuencia respiratoria/volumen tidal (f/Vt). Así mismo, la medición de la capacidad vital o la Pi-máx. para medir fuerza muscular es aplicado por $57,0 \%$ y respecto al uso de corticoides IV previo a la extubación $4,7 \%$ (10/214) expresaron nunca usarlos. Como conclusión no todos los terapistas respiratorios utilizan con igual frecuencia los criterios de destete.

Palabras clave: Destete, ventilación mecánica, prueba de respiración espontánea, extubación.

\section{ABSTRACT}

Weaning from mechanical ventilation is the process of disconnecting or removing artificial ventilatory support. There are no absolute criteria to prevent a failed weaning or without complications but it is advisable to use protocols with their predictors and weaning criteria to minimize unwanted consequences. The objective was to establish the knowledge, attitudes and practices of respiratory care professionals in the weaning of mechanical ventilation in public and private centers in the city of Guayaquil. The research design was quantitative, not experimental, transversal, observational, with a descriptive approach. The sample consisted of 214 respiratory therapists from 6 private clinics and 8 public hospitals in the city of Guayaquil to which a survey of sixteen questions was applied as a data collection instrument. As a result, it was obtained that $58 \%$ of therapists use weaning protocols, ensuring that $98 \%$ of cases are indicated by the doctor and only $2 \%$ perform weaning on their own decision. Regarding the evaluation of the hemodynamics of the patient, $88.3 \%$ of respiratory therapists expressed using the Tobin index, respiratory rate / tidal volume ratio ( $\mathrm{f} / \mathrm{Vt}$ ). Likewise, the measurement of the vital capacity or the Pimax to measure muscular strength is applied by $57.0 \%$ with respect to the use of IV corticosteroids before extubation 4.7\% (10/214) expressed never using them. In conclusion, not all respiratory therapists use the weaning criteria with equal frequency.

Keywords: Weaning, Mechanical Ventilation, Spontaneous breathing test, Extubation.

\section{RESUMO}

O desmame da ventilação mecânica é o processo de desconectar ou remover o suporte ventilatório artificial. Não há critérios absolutos para impedir o desmame com falha ou sem complicações, mas é aconselhável usar protocolos com seus preditores e critérios de desmame para minimizar consequências indesejadas. O objetivo foi estabelecer o conhecimento, atitudes e práticas dos profissionais de assistência respiratória no desmame da ventilação mecânica em centros públicos e privados da cidade de Guayaquil. O desenho da pesquisa foi quantitativo, não experimental, transversal, observacional, com abordagem descritiva. A amostra foi composta por 214 fisioterapeutas de 6 clínicas particulares e 8 hospitais públicos da cidade de Guayaquil, nos quais foi aplicado um questionário de dezesseis questões como instrumento de coleta de dados. Como resultado, obteve-se que $58 \%$ dos terapeutas utilizam protocolos de desmame, garantindo que $98 \%$ dos casos sejam indicados pelo médico e apenas $2 \%$ realizem o desmame por decisão própria. Em relação à avaliação da hemodinâmica do paciente, 88,3\% dos fisioterapeutas expressaram pelo índice de Tobin, razão frequência respiratória / volume corrente ( $\mathrm{f} / \mathrm{Vt}$ ). Da mesma forma, a medida da capacidade vital ou do Pimax para medir a força muscular é aplicada em 57,0\% no que diz respeito ao uso de corticosteroides intravenosos antes da extubação. Concluindo, nem todos os fisioterapeutas usam os critérios de desmame com igual frequência.

Palavras-chave: Desmame, ventilação mecânica, teste de respiração espontânea, extubação. 


\section{Introducción}

La ventilación mecánica (VM) es la sustitución artificial de la función pulmonar a través de respiradores. La VM tiene algunos objetivos fisiológicos, como lo son mantener y normalizar el intercambio gaseoso basado en una ventilación alveolar adecuada y mejorar la oxigenación arterial, reducir el trabajo respiratorio, así como clínicos: revertir la hipoxemia y la acidosis respiratoria aguda, aliviar la fatiga respiratoria, prevenir o revertir atelectasias, permitir la sedación o el bloqueo neuromuscular, y disminuir el consumo de oxígeno sistémico o miocárdi$\mathrm{CO}^{1}$.

Dado que la VM puede reemplazar por completo o parcialmente la respiración espontánea, se elige, según el tipo de soporte inspiratorio que ofrece la misma, el modo a utilizar considerando la patología o requerimientos de cada paciente. Una vez que el episodio que la requirió se ha resuelto es tiempo de realizar el destete. Es importante denotar que dentro del proceso de la ventilación mecánica también se debe tener presente el destete, el cual ocupa casi la mitad (40\%) del tiempo total de la ventilación mecánica. Hasta el año 2010, el porcentaje de tiempo dedicado al destete disminuyó en un $10 \%$ desde el cohorte realizado en $1998^{2}$.

El destete de la ventilación mecánica, conocido también como "weaning", es el proceso de desconexión o retirada gradual del soporte ventilatorio. Este procedimiento de desconexión del respirador busca que el paciente asuma de nuevo la respiración espontánea, y cubre el proceso de liberación del paciente del soporte mecánico y el tubo endotraquea| ${ }^{3,4}$.

Sin embargo, elegir el momento adecuado para el destete representa un desafío para los profesionales de la salud. Según Esteban et al, $75 \%$ de los pacientes ventilados son fáciles de destetar del ventilador con procesos simples, $10-15 \%$ de los pacien- tes requieren la utilización de un método de destete por 24 a 48 horas, 5-10\% requiere un destete gradual sobre un largo periodo de tiempo y tan sólo un 1\% depende crónicamente del respirador ${ }^{4}$.

Es de gran importancia clínica identificar el momento idóneo para extubar a un paciente ya que se debe encontrar un balance entre una extubación precoz o una prolongación innecesaria de la ventilación mecánica, debido a que ambas situaciones se encuentran relacionadas con un riesgo aumentado de complicaciones en pacientes críticamente enfermos ${ }^{4}$.

El proceso de destete de la ventilación mecánica comienza con el reconocimiento de la recuperación del fallo respiratorio agudo que tuvo como consecuencia la necesidad de iniciar la ventilación mecánica, o cuando la patología que llevó al uso del ventilador mecánico mejoró. Para evitar complicaciones indeseadas, existen criterios que ayudan a determinar el momento ideal para el retiro de la ventilación mecánica ${ }^{4,5}$.

La función muscular respiratoria además, depende de un adecuado aporte energético y un correcto aporte de oxígeno, por lo que el compromiso de sus determinantes como es el caso de la presencia de hipoxemia, de anemia, o una disminución del gasto cardíaco pueden comprometer la contractilidad muscular respiratoria, y disminuir su fuerza resistiva, al punto de fallar el destete ventilatorio, por lo que el Terapeuta Ocupacional en fin de reestablecer la funcionabilidad del cuerpo mediante un análisis del movimiento corporal para garantizar un adecuado aporte de oxígeno a los músculos respiratorios vigilando la oxigenación, los niveles de perfusión tisular y de hemoglobina, y como parte de un equipo interdisciplinario buscar las medidas necesarias para corregir el compromiso de éstos aspectos Los músculos de la respiración como son el diafragma y los intercostales, sometidos a periodos prolongados de ventilación mecánica ge- 
neralmente se encuentran bajo movimientos pasivos, los cuales favorecen la atrofia y la disminución de la contracción, facilitando la aparición de acidosis respiratoria y el aumento del trabajo ventilatorio.

Las patologías neurológicas y neuromusculares que comprometen la musculatura respiratoria son otra causa importante de atrofia muscular y debilidad de la musculatura respiratoria, estas pueden ser desarrolladas antes del ingreso a la $\mathrm{UCl}$ o desarrolladas dentro de esta, tales como la polineuropatía del paciente crítico. A mayor cantidad de tiempo de estancia en $\mathrm{UCl}$, es la probabilidad que el paciente desarrolle la polineuropatía del paciente crítico, en pacientes con estas condiciones se debe considerar la posibilidad de realizar un destete ventilatorio bien planeado antes de someterlos a la extubación o incluir la posibilidad de traqueostomía para favorecer el destete ventilatorio, esto genera que la valoración terapéutica sea constante y permita guiar la toma de decisiones en este aspecto. Sin embargo, es válido el desarrollo previo de programas de rehabilitación neuromuscular donde se logre mejorar la función con automatización de un adecuado patrón ventilatorio, optimizando la coordinación, disminuyendo la atrofia, la debilidad muscular y favoreciendo la conversión de fibras musculares tipo II a tipo I, para favorecer la resistencia muscular respiratoria.

Esto es posible gracias a la realización de maniobras de Reexpansión torácico a través de la movilización de miembros superiores y el posicionamiento, la reeducación diafragmática el fortalecimiento de los músculos abdominales y accesorios de la inspiración, así como del uso de estrategias de retroalimentación respiratoria gracias al mecanismo de biofeedback con la ayuda del ventilador, a través de un comando verbal, visual y táctil.

\section{Condiciones para la desconexión:}

- Relación PaO2/FiO2 2200 o SaO2 $\geq 90 \%$ con $\mathrm{FiO} 2 \leq 0,40$ y PEEP $\leq 5 \mathrm{cmH} 2 \mathrm{O}$.

- Estabilidad hemodinámica definida como ausencia de hipotensión clínicamente significativa o que no requiere fármacos vasoactivos o que requiere fármacos vasoactivos a dosis bajas (dopamina o dobutamina $<5 \mathrm{mg} / \mathrm{kg} / \mathrm{min}$.).

- Temperatura $\leq 38^{\circ} \mathrm{C}$.

- Hemoglobina $\geq 8 \mathrm{gr} / \mathrm{dl}$.

- Nivel de conciencia adecuado: definido como paciente que se encuentra despierto o que es despertado fácilmente.

- Tos adecuada/Reflejo tusígeno presente

- Ausencia de secreciones traqueo bronquiales excesivas

- Resolución de la fase aguda de la patología por la cual el paciente fue intubado

- Función pulmonar adecuada

- Frecuencia Respiratoria (FR)<35 Rpm

- Presión inspiratoria máxima $\leq-20--25$ $\mathrm{cmH} 2 \mathrm{O}$

- Volumen Corriente (VT) >5 mL/kg-1

- Capacidad Vital (CV)>10 mL/kg-1

- FR/VT <105 Rpm -1/L-13,4.

En la actualidad, no existe un consenso acerca de cuándo es el momento exacto para realizar el destete que nos prevenga de una extubación fallida o de una Re-intubación no deseada, ya que cada paciente varía sus necesidades según sus características basales, su patología y comorbilidades; sin embargo existen protocolos, parámetros y predictores que sirven como una guía para el profesional.

El objetivo del presente trabajo es determinar el nivel de conocimiento de los profesionales del cuidado respiratorio respecto al destete de la ventilación mecánica. Se busca determinar si ellos conocen las herramientas más utilizadas en el proceso del destete, los diferentes parámetros clínicos y de laboratorio, así como el tiempo utilizado en el mismo. 


\section{Materiales y métodos}

La investigación se realizó en 6 clínicas privadas y 8 hospitales públicos de la ciudad de Guayaquil, Ecuador, en un período de 2 meses en 2018, con una muestra de 214 terapistas respiratorios. La encuesta fue aplicada al azar a terapistas tanto de sexo masculino como femenino. No se incluyó en el estudio a estudiantes, licenciados en enfermería ni a médicos. El diseño del estudio es no experimental, transversal, observacional, y descriptivo. La recolección de los datos fue por medio de una encuesta escrita de dieciséis preguntas aprobada por expertos, aplicada a los 214 terapistas respiratorios con previo consentimiento in- formado. La información se procedió a almacenar y tabular los datos en Microsoft Excel y para cálculo de frecuencia se usó el programa de paquete estadístico Epidat.

\section{Resultados}

De Los 214 terapistas respiratorios encuestados, el 60,7\% (130/214) laboraban en un centro público y $39,3 \%(80 / 214)$ en centros privados, de los cuales el 58\% (124/214) manifestó el uso de protocolos al momento del destete de ventilación mecánica, asegurando que el 98\% (210/214) de los casos es indicado por el médico y solo el $2 \%$ realizan el destete por decisión propia (Tabla 1).

Tabla 1. Terapistas respiratorios en el destete de ventilación mecánica

\begin{tabular}{|c|c|c|c|}
\hline \multirow[b]{2}{*}{ Ítem } & \multirow[b]{2}{*}{ Descripción } & \multicolumn{2}{|c|}{ Frecuencia } \\
\hline & & $\mathrm{N}$ & $\&$ \\
\hline \multirow[t]{2}{*}{ Terapistas Respiratorios } & Públicos & 130 & 60,7 \\
\hline & Privados & 84 & 39,3 \\
\hline \multirow[t]{2}{*}{ Uso de Protocolo } & Sí & 124 & 58 \\
\hline & No & 90 & 42 \\
\hline \multirow[t]{2}{*}{ Destete } & Recomendado por el médico & 210 & 98 \\
\hline & Decisión del terapista & 4 & 2 \\
\hline
\end{tabular}

Fuente: Los autores

En cuanto a la evaluación de la hemodinámica del paciente, 88,3\% (189/214) de los terapistas respiratorios expresaron usar el índice de Tobin, cociente frecuencia respiratoria/volumen tidal (f/Vt), incluyendo aqueIla que incluso lo usan rara vez, solo $11,7 \%$ nunca han utilizado este índice. Así mismo, la medición de la capacidad vital o la Pi- máx. para medir fuerza muscular es aplicado por 57,0\% (122/214), de los cuales el $36,9 \%$ (79/214) pertenecen a terapistas del sector público. La presión soporte y PEEP (presión positiva al final de la espiración) previo a la colocación de T02 es utilizado siempre en el 52 , $8 \%$ de los casos (Tabla 2). 
Tabla 2. Evaluación hemodinámica del paciente

\begin{tabular}{|c|c|c|c|c|c|c|c|}
\hline \multirow[b]{2}{*}{ Ítem } & \multirow[b]{2}{*}{ Descripción } & \multicolumn{2}{|c|}{ Públicos } & \multicolumn{2}{|c|}{ Privados } & \multicolumn{2}{|c|}{ Total } \\
\hline & & $\mathrm{N}$ & $\%$ & $\mathrm{~N}$ & $\%$ & $\mathrm{n}$ & $\%$ \\
\hline \multirow{7}{*}{$\begin{array}{l}\text { ¿Ud. utiliza el índice } \\
\text { de Tobin? }\end{array}$} & Nuca & 16 & 7,5 & 9 & 4,2 & 25 & 11,7 \\
\hline & Rara vez & 15 & 7,0 & 16 & 7,5 & 31 & 14,5 \\
\hline & Frecuentemente & 18 & 8,4 & 5 & 2,3 & 23 & 10,7 \\
\hline & Casi siempre & 36 & 16,8 & 10 & 4,7 & 46 & 21,5 \\
\hline & Siempre & 31 & 14,5 & 35 & 16,4 & 66 & 30,8 \\
\hline & Sin respuesta & 14 & 6,5 & 9 & 4,2 & 23 & 10,7 \\
\hline & Total & 130 & 60,7 & 84 & 39,3 & 214 & 100 \\
\hline \multirow{7}{*}{$\begin{array}{c}\text { ¿Usted mide la } \\
\text { capacidad vital } \\
\text { o la Plmax para } \\
\text { medir fuerza } \\
\text { muscular? }\end{array}$} & Nuca & 13 & 6,1 & 6 & 2,8 & 19 & 8,9 \\
\hline & Rara vez & 25 & 11,7 & 31 & 14,5 & 56 & 26,2 \\
\hline & Frecuentemente & 6 & 2,8 & 2 & 0,9 & 8 & 3,7 \\
\hline & Casi siempre & 7 & 3,3 & 2 & 0,9 & 9 & 4,2 \\
\hline & Siempre & 79 & 36,9 & 43 & 20,1 & 122 & 57,0 \\
\hline & Sin respuesta & 0 & 0,0 & 0 & 0,0 & 0 & 0,0 \\
\hline & Total & 130 & 60,7 & 84 & 39,3 & 214 & 100 \\
\hline \multirow{7}{*}{$\begin{array}{l}\text { ¿Ud. prefiere utilizar } \\
\text { la presión soporte } \\
\text { y PEEP previo a la } \\
\text { colocación de T02? }\end{array}$} & Nuca & 3 & 1,4 & 2 & 0,9 & 5 & 2,3 \\
\hline & Rara vez & 2 & 0,9 & 2 & 0,9 & 4 & 1,9 \\
\hline & Frecuentemente & 20 & 9,3 & 8 & 3,7 & 28 & 13,1 \\
\hline & Casi siempre & 31 & 14,5 & 20 & 9,3 & 51 & 23,8 \\
\hline & Siempre & 66 & 30,8 & 47 & 22,0 & 113 & 52,8 \\
\hline & Sin respuesta & 8 & 3,7 & 5 & 2,3 & 13 & 6,1 \\
\hline & Total & 130 & 60,7 & 84 & 39,3 & 214 & 100 \\
\hline
\end{tabular}

PEEP: Presión Positiva al final de la Espiración. Plmax: Presión Inspiratoria máxima.

Fuente: Los autores

La prueba de fuga (Cuff-Leak Test) previo de la extubación es realizada siempre 29,4\% (63/214), Casi siempre 17,8\% (38/214), frecuentemente $136 \%(29 / 214)$ y rara vez $16,8 \%$ (36/214), solo $20,1 \%(43 / 214)$ manifestaron nunca haber hecho esta prueba. Respecto al uso de corticoides IV previo a la extubación 4,7\% (10/214) expresaron nunca usarlos (Tabla 3 ). 
Tabla 3. Prueba de fuga previo a extubación y uso de corticoides

\begin{tabular}{|c|c|c|c|c|c|c|c|}
\hline \multirow[b]{2}{*}{ Ítem } & \multirow[b]{2}{*}{ Descripción } & \multicolumn{2}{|c|}{ Públicos } & \multicolumn{2}{|c|}{ Privados } & \multicolumn{2}{|c|}{ Total } \\
\hline & & $\mathrm{N}$ & $\%$ & $\mathrm{~N}$ & $\%$ & $\mathrm{n}$ & $\%$ \\
\hline \multirow{6}{*}{$\begin{array}{c}\text { ¿Ud. realiza la prueba de } \\
\text { fuga } \\
\text { previo de la extubación? }\end{array}$} & Nuca & 28 & 13,1 & 15 & 7,0 & 43 & 20,1 \\
\hline & Rara vez & 22 & 10,3 & 14 & 6,5 & 36 & 16,8 \\
\hline & Frecuentemente & 22 & 10,3 & 7 & 3,3 & 29 & 13,6 \\
\hline & Casi siempre & 25 & 11,7 & 13 & 6,1 & 38 & 17,8 \\
\hline & Siempre & 33 & 15,4 & 30 & 14,0 & 63 & 29,4 \\
\hline & Sin respuesta & 0 & 0,0 & 5 & 2,3 & 5 & 2,3 \\
\hline & Total & 130 & 60,7 & 84 & 39,3 & 214 & 100 \\
\hline \multirow{7}{*}{$\begin{array}{c}\text { ¿En su área se administra } \\
\text { un } \\
\text { corticoide IV previo a la } \\
\text { extubación? }\end{array}$} & Nuca & 6 & 2,8 & 4 & 1,9 & 10 & 4,7 \\
\hline & Rara vez & 8 & 3,7 & 3 & 1,4 & 11 & 5,1 \\
\hline & Frecuentemente & 9 & 42 & 4 & 19 & 13 & 61 \\
\hline & Casi siempre & 45 & 21,0 & 15 & 7,0 & 60 & 28,0 \\
\hline & Siempre & 62 & 29,0 & 58 & 27,1 & 120 & 56,1 \\
\hline & Sin respuesta & 0 & 0,0 & 0 & 0,0 & 0 & 0,0 \\
\hline & Total & 130 & 60,7 & 84 & 39,3 & 214 & 100 \\
\hline
\end{tabular}

Fuente: Los autores

El criterio de destete mayormente usado por los terapistas es la valoración de la gasometría en $76,2 \%$ seguido del uso de la Pa08/Fi02 en 50,0\% (107/214), por otra parte, la valoración de la gasometría como criterio es usado en 76,2\% (163/214) de los casos. La invasión no invasiva como ayuda a extubación es usada rara vez en 30,8\% (66/214), el 55,1\% (118/214) indicó que rara vez sucede la Re-intubación en pacientes después de 48 o 72 horas extubado (Tabla 4).

Tabla 4. Frecuencia de programación de las extubaciones

\begin{tabular}{|c|c|c|c|c|c|c|c|}
\hline \multirow[b]{2}{*}{ Ítem } & \multirow[b]{2}{*}{ Descripción } & \multicolumn{2}{|c|}{ Públicos } & \multicolumn{2}{|c|}{ Privados } & \multicolumn{2}{|c|}{ Total } \\
\hline & & $\mathrm{N}$ & $\%$ & $\mathrm{~N}$ & $\%$ & $\mathrm{~N}$ & $\%$ \\
\hline \multirow{7}{*}{$\begin{array}{c}\text { ¿En su área las extubaciones } \\
\text { son programadas? }\end{array}$} & Nuca & 2 & 0,9 & 2 & 0,9 & 4 & 1,9 \\
\hline & Rara vez & 9 & 4,2 & 4 & 1,9 & 13 & 6,1 \\
\hline & Frecuentemente & 14 & 6,5 & 7 & 3,3 & 21 & 9,8 \\
\hline & Casi siempre & 22 & 10,3 & 14 & 6,5 & 36 & 16,8 \\
\hline & Siempre & 31 & 14,5 & 27 & 12,6 & 58 & 27,1 \\
\hline & Sin respuesta & 52 & 24,3 & 30 & 14,0 & 82 & 38,3 \\
\hline & Total & 130 & 60,7 & 84 & 39,3 & 214 & 100 \\
\hline \multirow{5}{*}{$\begin{array}{c}\text { ¿Con qué frecuencia en su } \\
\text { área } \\
\text { hay extubaciones no } \\
\text { programadas? }\end{array}$} & Nuca & 18 & 8,4 & 6 & 2,8 & 24 & 11,2 \\
\hline & Rara vez & 69 & 32,2 & 53 & 24,8 & 122 & 57,0 \\
\hline & Frecuentemente & 15 & 7,0 & 9 & 4,2 & 24 & 11,2 \\
\hline & Casi siempre & 21 & 9,8 & 10 & 4,7 & 31 & 14,5 \\
\hline & Siempre & 7 & 3,3 & 6 & 2,8 & 13 & 6,1 \\
\hline
\end{tabular}




\begin{tabular}{|c|c|c|c|c|c|c|c|c|}
\hline & Sin respuesta & & 0 & 0,0 & 0 & 0,0 & 0 & 0,0 \\
\hline & & Total & 130 & 60,7 & 84 & 39,3 & 214 & 100 \\
\hline \multirow{7}{*}{$\begin{array}{c}\text { ¿En su área colocan al } \\
\text { paciente en T02 } \\
\text { y después de } 4 \text { horas lo } \\
\text { extuban? }\end{array}$} & Nuca & & 15 & 7,0 & 17 & 7,9 & 4 & 15,0 \\
\hline & Rara vez & & 43 & 20,1 & 21 & 9,8 & 13 & 29,9 \\
\hline & Frecuentemente & & 24 & 11,2 & 15 & 7,0 & 21 & 18,2 \\
\hline & Casi siempre & & 25 & 11,7 & 20 & 9,3 & 36 & 21,0 \\
\hline & Siempre & & 23 & 10,7 & 11 & 5,1 & 58 & 15,9 \\
\hline & Sin respuesta & & 0 & 0,0 & 0 & 0,0 & 82 & 0,0 \\
\hline & & Total & 130 & 60,7 & 84 & 39,3 & 214 & 100 \\
\hline \multirow{7}{*}{$\begin{array}{l}\text { ¿En su área colocan al } \\
\text { paciente en T02 } \\
\text { y después de } 8 \text { horas lo } \\
\text { extuban? }\end{array}$} & Nuca & & 54 & 25,2 & 39 & 18,2 & 93 & 43,5 \\
\hline & Rara vez & & 34 & 15,9 & 26 & 12,1 & 60 & 28,0 \\
\hline & Frecuentemente & & 24 & 11,2 & 11 & 5,1 & 35 & 16,4 \\
\hline & Casi siempre & & 15 & 7,0 & 7 & 3,3 & 22 & 10,3 \\
\hline & Siempre & & 3 & 1,4 & 1 & 0,5 & 4 & 1,9 \\
\hline & Sin respuesta & & 0 & 0,0 & 0 & 0,0 & 0 & 0,0 \\
\hline & & Total & 130 & 60,7 & 84 & 39,3 & 214 & 100 \\
\hline
\end{tabular}

Fuente: Los autores

El criterio de destete mayormente usado por los terapistas es la valoración de la gasometría en $76,2 \%$ seguido del uso de la Pa08/Fi02 en 50,0\% (107/214), por otra parte, la valoración de la gasometría como criterio es usado en 76,2\% (163/214) de los casos. La invasión no invasiva como ayuda a extubación es usada rara vez en 30,8\% (66/214), el 55,1\% (118/214) indicó que rara vez sucede la Re-intubación en pacientes después de 48 o 72 horas extubado (Tabla 4).

Tabla 5. Uso de criterios de destete de los terapistas respiratorios

\begin{tabular}{clccccccc}
\hline & & \multicolumn{2}{c}{ Públicos } & \multicolumn{2}{c}{ Privados } & \multicolumn{2}{c}{ Total } \\
& \multicolumn{1}{c}{ Ítem } & Descripción & $\mathrm{n}$ & $\%$ & $\mathrm{~N}$ & $\%$ & $\mathrm{~N}$ & $\%$ \\
\hline & Nuca & 0 & 0,0 & 0 & 0,0 & 0 & 0,0 \\
& Rara vez & 9 & 4,2 & 6 & 2,8 & 15 & 7,0 \\
¿Ud. utiliza la Pa02/Fi02 & Frecuentemente & 7 & 3,3 & 4 & 1,9 & 11 & 5,1 \\
como criterio de destete? & Casi siempre & 36 & 16,8 & 32 & 15,0 & 68 & 31,8 \\
& Siempre & 70 & 32,7 & 37 & 17,3 & 107 & 50,0 \\
& Sin respuesta & 8 & 3,7 & 5 & 2,3 & 13 & 6,1 \\
\hline & & Total & 130 & 60,7 & 84 & 39,3 & 214 & 100 \\
\hline \multirow{2}{*}{ ¿Ud. valora la gasometría } & Rara vez & 1 & 0,5 & 1 & 0,5 & 2 & 0,9 \\
& Frecuentemente & 4 & 1,9 & 2 & 0,9 & 6 & 2,8 \\
& Casi siempre & 20 & 9,3 & 14 & 6,5 & 34 & 15,9 \\
& Siempre & 100 & 46,7 & 63 & 29,4 & 163 & 76,2 \\
& Sin respuesta & 5 & 2,3 & 4 & 1,9 & 9 & 4,2 \\
\hline & \multicolumn{2}{c}{ Total } & 130 & 60,7 & 84 & 39,3 & 214 & 100 \\
\hline
\end{tabular}




\begin{tabular}{clcccccc} 
& Nuca & 14 & 6,5 & 10 & 4,7 & 24 & 11,2 \\
& Rara vez & 38 & 17,8 & 28 & 13,1 & 66 & 30,8 \\
\multirow{2}{*}{$\begin{array}{c}\text { ¿Ud. utiliza Ventilación No } \\
\text { Invasiva }\end{array}$} & Frecuentemente & 34 & 15,9 & 12 & 5,6 & 46 & 21,5 \\
como ayuda a la extubación? & Casi siempre & 30 & 14,0 & 31 & 14,5 & 61 & 28,5 \\
& Siempre & 14 & 6,5 & 3 & 1,4 & 17 & 7,9 \\
& Sin respuesta & 0 & 0,0 & 0 & 0,0 & 0 & 0,0 \\
\hline \multirow{2}{*}{$\begin{array}{c}\text { ¿Cuál es la incidencia de } \\
\text { reintubación }\end{array}$} & Nucalal & 130 & 60,7 & 84 & 39,3 & 214 & 100 \\
\hline en su área después de 48 o 72 & Rara vez & 20 & 9,3 & 8 & 3,7 & 28 & 13,1 \\
horas & & 70 & 32,7 & 48 & 22,4 & 118 & 55,1 \\
de extubado el paciente? & Frecuentemente & 18 & 8,4 & 12 & 5,6 & 30 & 14,0 \\
& Casi siempre & 9 & 4,2 & 8 & 3,7 & 17 & 7,9 \\
& Siempre & 5 & 2,3 & 3 & 1,4 & 8 & 3,7 \\
& Sin respuesta & 8 & 3,7 & 5 & 2,3 & 13 & 6,1 \\
\hline
\end{tabular}

Fuente: Los autores

\section{Discusión}

El uso de protocolos es superior a una decisión unipersonal tomada junto a la cama del enfermo y es indispensable aplicarlo para lograr un destete exitoso6, los resultados de las encuestas realizadas demuestran que no existe uniformidad en la metodología a seguir para realizar el destete en el paciente que se somete a ventilación mecánica ya que solo el 58\% manifestó el uso de protocolos al momento del destete de ventilación mecánica, asegurando que el $98 \%$ de los casos es indicado por el médico.

El destete debe cumplir condiciones para efectuarlo con éxito, aplicando los predictores más adecuados según el tipo de paciente. El Consenso Brasileño del 2008, señala como mejores predictores de éxito: el esfuerzo respiratorio negativo, la presión inspiratoria máxima, la ventilación minuta y la relación de la presión de oclusión de la vía aérea y el índice de CROP (complacía, presión inspiratoria máxima, frecuencia respiratoria y oxigenación), siendo los dos últimos los de mayor aplicación clínica?.

Investigaciones afirman que existen múl- tiples índices predictivos de la evolución de la desconexión de ventilación mecánica, uno de los más difundidos es el que relaciona frecuencia respiratoria y volumen corriente (f / VT) o Índice de Tobin7, 8 y 9, coincidiendo con los resultados del estudio donde $88,3 \%$ de los terapistas respiratorios expresaron usar el índice de Tobin, cociente frecuencia respiratoria/volumen tidal ( $f$ / Vt). Así mismo, la medición de la capacidad vital o la Pi-máx. para medir fuerza muscular es aplicado por 57,0\%.

La presión soporte y PEEP (presión positiva al final de la espiración) previo a la colocación de T02 es utilizado siempre en el 52, 8\% de los casos, el concepto de PEEP ha evolucionado a lo largo de los años desde que se describió por primera vez en 196510. Suter et al., describen la mejor PEEP como el nivel de PEEP necesario para conseguir mejor transporte de oxígeno11. Recientemente, se concluyó que el mejor PEEP es aquella que permita unos niveles adecuados de $\mathrm{PaCO} 2$ con una $\mathrm{FiO} 2$ inferior a 0,512. El aumento de PEEP por encima de estas necesidades puede resultar nocivo, ya que repercute en el sistema cardiovascular y pulmonar aumentando la presión in- 
tratorácica y la resistencia pulmonar, por tal causa la importancia de medir PEEP previo a la colocación de T02 es elevada.

La prueba de fuga (Cuff-Leak Test) previo de la extubación es realizada siempre $29,4 \%$, Casi siempre 17,8\%, frecuentemente $13,6 \%$ y rara vez $16,8 \%$, solo $20,1 \%$ manifestaron nunca haber hecho esta prueba. Investigaciones recomiendan usar la prueba de fuga antes de la extubación13 y 14, ya que es considerada como una variable de potencial factor de riesgo para el desarrollo de edema glótico o subglótico. Su ausencia (prueba de fuga positiva) implica un alto riesgo de obstrucción de la vía aérea superior, pero a su vez su presencia no la descarta. En el metaanálisis consultado, la prueba demostró tener mayor precisión para detectar obstrucción de la vía aérea superior [predicción de edema laríngeo: OR 18.78 (9 estudios)] que para predecir el riesgo de Re-intubación [predicción de Re-intubación: OR 10.37 (3 estudios)] secundaria a obstrucción de la vía aérea superior. La ausencia de fuga aumenta la probabilidad de Re-intubación a 17\% y la presencia de la fuga disminuye la probabilidad de Re-intubación a un 2\% ${ }^{15}$. Respecto al uso de corticoides IV previo a la extubación el $95,3 \%$ indicaron usarlo y solo 4,7\% expresaron nunca usarlos, esto podría inferirse que los terapistas conocen los beneficios que aporta la administración profiláctica de corticoesteroides previo a la extubación para disminuir los casos de edema laríngeo y obstrucción aérea alta ${ }^{16,17}$.

Las extubaciones son programadas en $98,1 \%$ de los casos, incluyendo las respuestas para siempre, casi siempre, frecuentemente y rara vez, solo 1,9\% (4/214) nunca realizan las extubaciones de manera programada coincidiendo con el estudio de Boles et al., donde la incidencia de las extubaciones no planeadas varía de $0.3 \%$ a $16 \%{ }^{3}$. Por otra parte $29,9 \%$ colocan al paciente rara vez T02 y después de 4 horas lo extuban.
El criterio de destete mayormente usado por los terapistas es la valoración de la gasometría en $76,2 \%$ seguido del uso de la $\mathrm{Pa08/}$ Fi02 en 50,0\%, por otra parte la valoración de la gasometría como criterio es usado en $76,2 \%$ de los casos. La invasión no invasiva como ayuda a extubación es usada rara vez en $30,8 \%$, el $55,1 \%$ indicó que rara vez sucede la Re-intubación en pacientes después de 48 o 72 horas extubado. A esta Re-intubación luego de las 48-72 horas se la conoce como destete fallido o fracaso del destete ya que el destete exitoso ocurre cuando se ha llevado a cabo la extubación y hay ausencia de soporte ventilatorio por más de 72 horas. Las causas más comunes del fracaso del destete son: alteraciones del intercambio gaseoso, fracaso de los músculos respiratorios, factores cardiovasculares, y factores no cardiopulmonares. La actitud más importante para lograr un destete exitoso es la evaluación diaria holística del paciente con sus respectivos parámetros y la actuación precoz ${ }^{18}$.

\section{Conclusiones}

Los parámetros clínicos, fisiológicos y de laboratorio que indican una posible preparación para el destete exitoso deben continuar siendo evaluados sistemática y diariamente para seleccionar a los candidatos idóneos para el proceso de retirada de la ventilación mecánica. Este proceso debe iniciarse tan pronto el paciente esté listo para así acortar tiempo de ventilación mecánica innecesaria así y disminuir sus complicaciones como por ejemplo la neumonía asociada al ventilador. Bajo estas recomendaciones se espera que en un futuro el tiempo dedicado al destete continúe disminuyendo y aumentando los destetes exitosos.

\section{Bibliografía}

1. Slutsky AS. Mechanical ventilation. American College of Chest Physicians' Consensus Conference. Chest. diciembre de 1993; 104(6):1833-59.

2. Esteban A, Frutos-Vivar F, Muriel A, Ferguson ND, Peñuelas $\mathrm{O}$, Abraira $\mathrm{V}$, et al. Evolution of Mortality

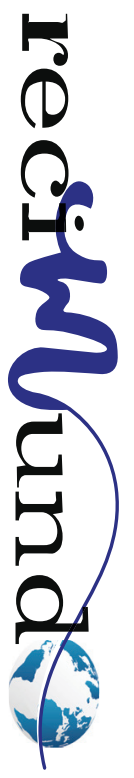


over Time in Patients Receiving Mechanical Ventilation. 2013; 188(2):220-30.

3. Boles J-M, Bion J, Connors A, Herridge M, Marsh B, Melot C, et al. Weaning from mechanical ventilation. Eur Respir J. el 30 de abril de 2007; 29(5):1033.

4. Penuelas O, Thille AW, Esteban A. Discontinuation of ventilatory support: new solutions to old dilemmas. CurrOpinCritCare. febrero de 2015; 21(1):7481.

5. Esteban A, Alia I, Ibanez J, Benito S, Tobin MJ. Modes of mechanical ventilation and weaning. A national survey of Spanish hospitals. The Spanish Lung Failure Collaborative Group. Chest. octubre de 1994;106(4):1188-93.

6. García VE. Ventilación no invasiva tras el fracaso en el destete. Rev Electr Med Intens. 2008; 8: 1-4

7. Caballero A. Ventilación artificial. Conceptos básicos. En: Caballero López A, Hernández H, editor. Terapia Intensiva. La Habana: Ciencias Médicas; 1988: 467-536.

8. Yang KL, Tobin MJ. A prospective study of indexes predicting the outcome of trials of weaning from mechanical ventilation. $\mathrm{N}$ Engl $\mathrm{J}$ Med. el 23 de mayo de 1991; 324(21):1445-50.

9. Bevilacqua C. Destete de ventilación mecánica. RAMR. 2016; 3: 286-287.

10. Coplin WM, Pierson DJ, Cooley KD, Newell DW, Rubenfeld GD. Implications of Extubation Delay in Brain-Injured Patients Meeting Standard Weaning Criteria. 2000; 161(5):1530-6.

11. Suter PM, Fairley HB, Isenberg MD. Optimum end expiratory airway pressure in patients with acute pulomonary failure. N Engl J. 1975; 292 (6): 284-289.
12. Briel M, Meade M, Meract A, Brower RG, Talmor D, Walter SD et al. Higher vs lower positive end expiratory pressure in patients with acute lung injury and acute respiratory distress syndrome: systematic reiew and meta-analysis. JAMA. 2010; 303 (9): 865-873.

13. Miller RL, Cole RP. Association between reduced cuff leak volume and postextubation stridor. Chest 1996; 110(4): 1035-1040.

14. Chung YH, Chao TY, Chiu CT, et al. The cuff-leak test is a simple tool to verify severe laryngeal edema in patients undergoing long-term mechanical ventilation. Crit Care Med 2006; 34 (2): 409-414.

15. Ochoa ME, del Carmen Marín M, Frutos-Vivar F, Gordo F, Latour-Pérez J, Calvo E, et al. Cuff-leak test for the diagnosis of upper airway obstruction in adults: a systematic review and meta-analysis. Intensive Care Med. 2009; 35(7):1171.

16. Fan $T$, Wang G, Mao B, Xiong Z, Zhang Y, Liu $X$, et al. Prophylactic administration of parenteral steroids for preventing airway complications after extubation in adults: meta-analysis of randomised placebo controlled trials. BMJ [Internet]. el 20 de octubre de 2008; 337. Disponible en: https://www. bmj.com/content/337/bmj.

17. Thille AW, Richard J-CM, Brochard L. The decision to extubate in the intensive care unit. Am J Respir Crit Care Med. el 15 de junio de 2013; 187(12):1294-302.

18.Frutos F, Alia I, Lorenzo MI, García Pardo L, Nolla M, Ibáñez J. Utilización de la ventilación mecánica en 72 unidades de Cuidados Intensivos en España. Med Intens. 2003; 7(01):1-12.

\section{CITAR ESTE ARTICULO:}

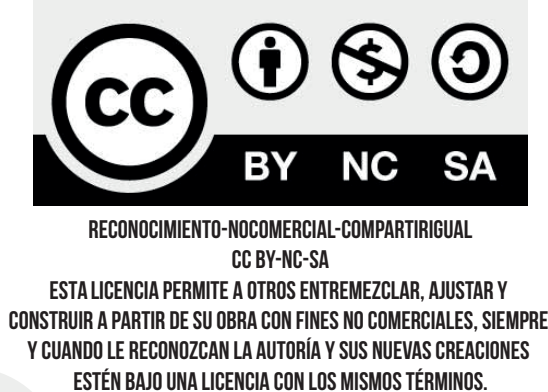

Castro García, R., Pazmiño Chancay, M., Zambrano Iza, M. \& Sánchez Acuña,

E. (2020). Destete de la ventilación mecánica, Guayaquil 2019. RECIMUNDO,

4(3), 42-52. doi:10.26820/recimundo/4.(3).julio.2020.42-52 